\title{
Research of the Method for EEG Source Localization
}

\author{
Qiu Lijun ${ }^{1}$, Liu Wenqiang ${ }^{2}$, Fan Qifu ${ }^{3}$, Liu Huan ${ }^{4}$ \\ College of control engineering, Xijing University, Xi'an 710123 \\ E-mail:1qiulij@fmmu.edu.cn; 2Liu-wq-66@163.com
}

Key Words: EEG forward problem, EEG inverse problem, Current dipole, Soucre localization Abstract: This paper describes the advantages and disadvantages of various models to establish methods and EEG localization problem in the head model used and the source model, focusing on research and analysis of the latest developments to solve the problem of EEG localization algorithm principle, and finally introduced to solve EEG inverse problem development direction.

\section{Introduction}

Human brain in the process of thinking will produce electromagnetic fields, these electromagnetic fields called EEG. Two key issues in the study of EEG are EEG forward problem and EEG inverse problem. Positive problem is that the distribution of known sources, to solve the electric field intensity generated by the scalp, inverse problem, also known as the brain power source localization, it is the electric field strength value from the scalp observation to reverse the information of the power of the brain activity. Brain source localization can obtain the information of the brain's internal nerve activity source without trauma, so as to analyze the function of Pathology, physiology and psychology. In addition, according to the EEG response to different stimuli can determine the location of brain function or even partition range, and then used to explore the brain activity patterns senior.

\section{Model EEG Problem}

Suppose model $m$ observation points outside the brain detects brain electric field value $V=\left(V_{1}, V_{2}, \mathrm{~L}, V_{m}\right)^{T}$ of $n$ EEG excitement generated source $P=\left(p_{1}, p_{2}, \mathrm{~L}, p_{n}\right)^{T}$, using the system's point of view to get EEG problem is as follows:

$$
V(t)=G(t) P(t)+N(t)
$$

Wherein, t represents the time, $N=\left(N_{1}, N_{2}, \mathrm{~L}, N_{m}\right)^{T}$ represents the noise, and $G$ represents the transfer function matrix.

Currently on brain power positioning of research, mainly problem is in the choice of the model, source localization method and localization accuracy of other cutting-edge topics. Selecting the source model problems and head model to solve the problem plays a very important role, in 1969, Plnosye proved biological electromagnetic study, the brain was seen as a closure of the electromagnetic system, its law suit can be used quasi-static approximation the Maxwell equations described as follows [4]:

$$
\left\{\begin{array}{c}
E=-\nabla V \\
\nabla \times B=\mu_{0} J \\
\nabla \cdot B=0 \\
\mathrm{v} \mathrm{V}^{i}+\sigma E
\end{array}\right.
$$

Wherein, $\stackrel{v}{E}$ represents electric field, $V$ represents potential, $\stackrel{\vee}{B}$ represents Magnetic flux density, $\sigma$ represents conductivity, $\mu_{0}$ represents permeability, $\vec{J}$ represents total current density, $J^{i}$ represents primary current density, $\sigma \stackrel{V}{ }$ represents volume current density. Equation (2) can be determined by equation (1) information of $G, P$ for equation (1) to solve, namely EEG forward problem solving. 


\section{EEG forward problem}

EEG forward problem include the establishment of numerical calculation and brain electric field analysis model, in particular the distribution of known sources, solving the electric field intensity produced by the scalp. Current research in brain activity commonly used as equal current dipole (ECD) mathematical model, The basic idea is to ECD as a source, the brain of a relatively small area of concentrated electrical activity is an ECD simulation scalp EEG generated by this small area contains the location and intensity of information sources on the equivalent ECD, ECD final information can provide it represents the brain tissue. Source model has been used are: single-ECD model, multi-ECD model, instantaneous state of ECD model, time-weighted space ECD model, Pasual-Marqui, who made heavy minimize the use of three-dimensional mesh method, etc[1]. By volume conductor head model to simulate head model has been used are: ball-model, ellipsoid-model, concentric spherical model and real head model[2-4].

EEG forward problem calculation two method for solving: analytical and numerical methods for solving method. Analytical solution method focuses on the calculation method of the series solution has made new efforts in the 1950s Wilson, who first derived formulas Homogeneous Conducting Sphere in ECD potential, Frank gives a more concise form ECD, Brody etc. in 1973, lead-based field concept derived strictly for the ball on the surface of the potential of each point is calculated. At home, Yao Dezhong from Legendre ECD potential series expansion formula, discusses a potential solution satisfies the boundary conditions, we have made this issue the general analytical solution[5]. Foreign scholars in recent years, based on the series expressed in a variety of special functions in depth studies have been related to the anisotropy of multilayer balls, multi-layer ellipsoid situation, but due to the presence of series solution of precision and truncated on the practical application error problem. Therefore, further development of more and more rational analytical solution, but the solution is made in the calculation method of the new series of efforts is necessary.

Numerical method focuses on construction update to improve the positioning accuracy by applying different numerical methods, closer to the real situation of the head model and the development of appropriate algorithms. Commonly used methods are: boundary element method(BEM), finite element method(FEM), finite volume methods (FVM), and so on. He uses BEM to study the skull cavity position on the measuring electrode and its positioning on the ECD and the head table relationships[6]; Ferguson and other studies of the actual impact of the first type, but local changes in circumstances and EEG homogeneous medium organization, head shape, skull and scalp for EEG [7]. Awada compared two popular FEM calculation methods in different head model, a different way when the grid efficiency and accuracy[8]; Bradley and other applications of BEM and FEM method combine together to achieve in the case takes up less CUP resources made high accuracy and shows good convergence [9]; Sun and other applications of the artificial neural network model to FEM head significantly improves the accuracy and efficiency of the FEM calculations[10]. Mosher and other presents a Solving convenient simplified EEG forward problem analytical and numerical solutions of unified approach for EEG inverse problem [11]. The basic idea is that the solution of the direct problem is decomposed into the field of nuclear, depending on the geometry of field nuclear and the position and form of the dipole moment of the product's source and sensors.

\section{EEG inverse problem}

EEG inverse problem is internal electromagnetic activity measurement data reconstruction based on scalp electric field strength, the inverse problem is the non-unique theoretical difficulty Problems. In 1853, Helmholz proved that there can be many solutions on endogenous distribution corresponding to a given field distribution in vitro. Another difficulty is that the inverse problem of pathological features of EEG inverse problem, because the non-continuity between the observation space and solution space mapping, the inverse problem in the sense of Hadamard is ill-posed, showing strong pathological numerical algorithm, even little noise can cause instability and oscillation solution, while increasing the number of parameters with the solution of this morbid trend upward. Secondly, the measurement data also increases the incompleteness of the non-uniqueness of the solution of difficult to solve. Therefore, 
we must structure and the source of the solution space plus a variety of space and time limitations in solving the introduction of a variety of prior knowledge to be incorporated into a variety of meaningful constraint and numerical guidelines, so ill-posed problem of order the only solution to get within a certain range, or some sense justified. Thus when constructing numerical algorithms require careful consideration be given full expression in the form of solving problems and regularization policies.

\section{Optimization Methods}

At home and abroad, there are two main approaches to the study of EEG inverse problem: based on the nonlinear parameter inversion method ECD models and voxel imaging (VI) method based on current distribution model for general medical imaging.

\section{Based on nonlinear parameter inversion method ECD model}

Inversion algorithm based ECD model follows the main idea is to adjust the parameters of the forward ECD calculated results with the measured data fitting, because the problem itself does not apply to the non-uniqueness of qualitative reconciliation, this parameter is generally more difficult adjustment process, by its very nature on the reverse is a nonlinear optimization problem. Unknown sources are available in a limited parameter representation, that by constructing the objective function (generally based on least squares criterion), the specific operation according to equation (1) the objective function is as follows:

$$
J=\|\hat{V}-V\|_{F}^{2}
$$

Wherein, $\|\left.\mathrm{d}\right|_{F} ^{2}$ represents Frobenius norm, $\hat{V}$ represents electric field sensor value measured outside the brain, $V$ represents using ECD model's parameter calculation equation (1) the electric field theoretical value [12]. Results calculated parameters and the quasi goodness (GOF) on the physiological significance judge, GOF is defined as

$$
G o F=\sqrt{1-\frac{\|\hat{V}-V\|_{F}^{2}}{\|\hat{V}\|_{F}^{2}}}
$$

GOF values closer to 1 indicating a reasonable algorithm[6].

At present, algorithm based on ECD model at home and abroad have numerical methods and signal processing methods, most algorithms in an iterative manner. Local optimization algorithm steepest descent method, Levenberg-Marquardt method, Powen method, variable metric method for the deterministic algorithms, we can solve for ECD parameters, but the result depends on the initial value of the Select [13]. Global optimization methods clustering method [14], simulated annealing method [15], genetic algorithm (GA) [16], the evolution of learning is also used for EEG inverse problem.Different from the deterministic algorithm, they usually calculate the value of the objective function in a series of randomly selected points, so as to avoid the local convergence and improve the possibility of reaching the global minimum. Uutela, respectively, the above three kinds of global optimization methods of the calculation results were compared, the conclusion of the GA efficiency is the highest, but more time-consuming calculation [17].

Xie Yong, nonlinear inversion method based ECD model, the first application of differential evolution (DE) algorithm, the single dipole source reconstruction, the results show the DE algorithm is simple, accurate positioning advantages [18].

With the introduction of time - space ECD model will help to reduce the complexity of nonlinear optimization, the basic idea: assuming the existence of one or several ECD, its spatial position and orientation is kept constant, strength is as a function of time. The model was evoked potentials in the whole band by minimizing the least squares fit residuals. Since taking the observational record period of time, the use of a more complete model, fully utilizing the correlation to improve the positioning accuracy of the data, while the inferred behavior of dynamic evolution of EEG. 
Modern signal processing methods have been applied to the study of EEG source localizing and achieved good results have multiple signal classification (MUSIC) method and independent component analysis (ICA) method. Mosher first proposed the study [19] the subspace decomposition algorithm for EEG inverse problem with a dipole source to scan the entire distribution area, to determine the location of the dipole a generalized extreme value by finding generalized eigenvalue. Sekinhara, who studied the different sensory stimuli, among active sources produced when the brain is statistically independent, the MUSIC method can also be used to distinguish between them and positioning. China's Yao Dezhong established a method based on a multidimensional MUSCI delayed correlation matrices and experimental simulation study and actual data have achieved good results [20].Mosher and Leahy further development of the algorithm, we propose a recursive MUSIC (R-MUSCI) method [21], they took advantage of subspace correlation coefficient concepts, successfully resolved the judge dipole in three-dimensional space when searching for multiple extreme points, so that each time searching for just an extreme point, and synchronization source localization problem is solved.

ICA is the recent multi-channel signal processing method developed by blind source separation technology, Leonid Zhukov, who will be the maximum entropy ICA algorithm is introduced into a separate dipole source localization in time and space, the basic idea is to multi-channel EEG observations in accordance with the principle of statistical independence through the optimization algorithm is divided into several independent components to achieve enhancement and analysis of the signal, and then were localizing for each individual source.

\section{VI method based on the current distribution model}

Signal Space Projection(SSP) is applied to a recent study of EEG signal processing method, the basic idea is that the electric field value $\mathrm{M}$ measured electric field detector is considered M-dimensional vector $\mathrm{B}$, by such vector generation $\mathrm{M}$-dimensional space called signal space. If only one source activity (eg some interference source) at a time, measure the field vector of the source, then the other times, the problem of space research to the above sub-vertical vector space, then this subspace in completely including the source of information By using the SSP method to solve EEG inverse problem, it can eliminate the influence of some interference sources, and improve accuracy of localization [22]. Xie Yong and other using GA and DE algorithm for head EIT imaging study, the DE algorithm was used for the first time in EIT imaging with GA algorithm is compared, the DE algorithm is simple, rapid convergence and high precision reconstruction advantages.

\section{Other algorithm for EEG inverse problem EEG}

Nonlinear parameter inversion in many fields is a challenging task, the traditional method of nonlinear optimization algorithm combined with today's new global optimization, may be a better research direction. For example, the combined Guass-Newton method and quasi-Newton method, based on the results of each search iteration is determined using the former method or the latter method. Because the numerical calculations for large residual problem, quasi-Newton method has obvious advantages, it can speed up the convergence, but for a small residual problem, as long as the use of a simple algorithm can Guass-Newton method. In the GA search process of local optimization methods for the integration of thought, constitute a GA algorithm is to improve operational efficiency and quality of an effective means to solve, but now has many applications in EEG inverse problem on has yet to see the reports, it is worth noting that research methods. Neural network method developed in recent years, its main advantage is to start training Once a network, you no longer need to process complex calculations or iterations. Because in practice does not involve the forward calculation, in equivalent circumstances you can use any source or advanced head model. In addition, the neural network can capture the source localization question nonlinear dynamic information, while maintaining the EEG analysis necessary for robust noise, but the neural network also have global problems of slow algorithm.

Yao D proposed source mapping equivalent charge layer model for EEG achieve better results $[14,8]$. Chang-HwanIm and other proposed a constraint-based positioning method anatomical regions of the brain power, the method using a number of parameters to represent the spatial location of EEG, 
and applied to simulate EEG epilepsy patients [22,17]. In China, Zhao Shuang, the electrical dipole location problem presents a new theoretical model, the use of matrix theory positioning of multiple dipoles were analyzed, lists multiple dipole source localization matrix equations expression [19]. Li Jianwei proposed iterative approximation based on least squares multidimensional Variable Weight SVR is also an effective numerical solution of EEG localization, training accuracy according to brain source localization accuracy.

\section{Conclusion}

EEG source localization is an important aspect of cognitive science and brain damage brain lesion localization studies, is considered to be one of several important theoretical topic of the 21st century in the field of electronics, has important scientific significance and significant engineering the value of its research will help promote brain function of electromagnetic technology and functional brain imaging techniques combined. To further explore the brain information transmission and processing way, so as to provide for scientific, intelligent science and information science development life new inspiration, promote information science, cognitive crossover and Fusion and life sciences, as well as lesions accurately locate targets, and the current international concern stereotactic and functional neurosurgery have a positive role in promoting.

This paper reviews the methods commonly used in EEG localization of head model and source model, focusing on the latest developments in this field in recent years, the source location algorithm, introduced a new method of EEG inverse problem solving. Clear binding electromagnetism, applied mathematics to the study of localization, and borrowed from fluid mechanics, geophysics and atmospheric sciences applications such as numerical calculation method, absorbing some of the best algorithm is an important and effective way, but also our under the direction of research.

\section{Acknowledgements}

This work was financially supported by the science foundation research project of Xijing University (Grant No. XJ150112), the nature science foundation research project of Shaanxi province (Grant No. 2014JM4176) and the nature science foundation research project of Shaanxi province (Grant No. 2016JM1032).

\section{References}

[1] Zou L."Brain source localization based on nonlinear optimization and Independent Component Analysis". Zhejiang University.2004.

[2] He J. "MEG、 EEG Forward Problem Numerical Simulation and Inverse Problems Study". Guizhou University.2007.

[3] Zhang YC, Zou L, etc. Models and algorithms EEG Forward Problems. Journal of Biomedical Engineering,2014,21(2), pp.337-339.

[4] Xie YQ, Yuan JS, Ma XS, etc. "Calculation of EEG Problems with Aniso-rtopic Conducting Media by the Finite Volume Method". IEEE Trans Magnetics,2001, 37(5) pp.37-49.

[5] Zhang YC. "Application of FEM in the three-dimensional anisotropic EEG forward problem and cortical imaging techniques". Zhejiang University.2014.

[6] J.C.Mosher etc. "EEG and MEG: Foward Solution Inverse Methods". IEEE Trnas BME.1999,46(3), pp.245-259.

[7] Li Y. "Numerical calculation method for EEG inverse problem solving". Hebei University of Technology.2003.

[8] Cuffin B N. "A method for localizing EEG source in realistic head models". IEEE Trans.on BME,1995,42(1), pp.68-71. 
[9] Yao DZ, Rao N, etc. "Delay Subspace EEG inverse problem decomposition algorithm". Journal of Electronics,2000,28(4), pp.135-138.

[10]Yao DZ. Based on high order cumulant EEG inverse problem" Journal of Biomedical Engineering,2010,17(2), pp.174-178.

[11]Yao DZ, Zhou YC, etc. "A new method for EEG inverse problem: delays related MUSIC multidimensional method". Journal of Electronics,2001,29(4), pp.522-525.

[12]Xie Y, Xu JX, etc. "Phase space reconstruction of ECoG time sequences and extraction of nonlinear characteristic quantities". Journal of Physics.2002(2), pp.205-214.

[13] Hu J, etc. "MEG source localization using nonlinear optimization methods". Zhejiang University of Technology,2002.02(1), pp.9-12.

[14]Zhu HY,etc. "Realistic head shape model of multiple current dipole MEG source localization". Journal of Physics.2012, 10(10), pp.2393-2397.

[15]Liu XC. "Brain source localization of electromagnetic fields and MEG". Guizhou University.2007.

[16]Li JW,Wang YH, etc. "EEG source localization based on multi-dimensional output support vector regression". Chinese Journal of Clinical Rehabilitation Tissue Engineering,2009.4(17), pp.3256-3259.

[17]Wu CQ, Zhao S. "Research on electric dipole source localization problem". Journal of Physics.2007.09(56), pp.5180-5184.

[18]Wu CQ, Zhao S. "Multi- dipole source localization problem matrix analysis". Journal of Beijing Jiaotong University.2008.06(3), pp.56-59.

[19]Ma JM, Wang B, etc. "Based on chaos optimization algorithm multiple signal classification MEG source localization method". Journal of Biological and Physics. 2015.10(25), pp.359-363.

[20]Uutela K Hamalainen M,Salmelin R.Global optimization in the localization of neuromagnetic sources IEEE Tmns.on BME,1998,45(6):716-723.

[21]Miga M 1,Kerne T E,Darcey TM.Soucre localization using a current-density minimization approach. IEEE Trans.on BEM,2002,49(7):743-745.

[22]Cuffin B N. Effects of head shapes on EEGs and MEGs. IEEE Trans BME, 1990,37(11):15. 